\title{
A FULL-ORDER, ALMOST-DETERMINISTIC OPTICAL MATCHING ALGORITHM
}

\author{
Yu-Chiu Chao, Thomas Jefferson National Accelerator Facility, Newport News, VA 23606
}

\begin{abstract}
An algorithm was developed for beam envelope matching with fixed geometry, taking into account the full order behavior of the quadrupoles and their compounded effects. In the present approach an algorithm was employed to systematically reduce the overall dimensionality of the problem. This resulted in two algebraic systems that are independent but can be solved in tandem. At the numerical level these 2 reduced systems are input to an equation solver in Mathematica 4.0 based on Gröbner-basis techniques, capable of providing global solutions to such algebraic systems. This has resulted in a robust and effective algorithm for on-line optical beam envelope matching. The global nature of the algorithm also may point to possible deficiencies in the optical system configuration. An on-line high precision transfer matrix measurement program was developed in conjunction to provide input to this algorithm. Preliminary on-line testing on the CEBAF accelerator has positively demonstrated the effectiveness of this method.
\end{abstract}

\section{INTRODUCTION}

Exact global solutions to thick quadrupole lens matching with fixed geometry can offer accelerator design and control much more than is possible with local methods. The algebraic structure of the problem is highly complex. Numerical methods, on the other hand, cannot kick in too early before sufficient simplification has been made on this structure to make the calculation viable at all. Of key importance is the ability to reduce the dimensionality of the problem to the point where robust and efficient numerical algorithms can be called upon to obtain exact, global solutions. An algorithm [1] has been developed to systematically dimension-reduce similar but simpler problems to the point where closed form algebraic solutions can be obtained. In the current case a similar algorithm was applied to reduce the apparent 4dimensional problem, for which robust numerical solutions are not always guaranteed, to 2-dimensional ones, significantly enhancing the possibility of robust and efficient numerical solutions, including global ones. In this report the dimension reduction method and outcome, the numerical tool for obtaining global solutions, adaptation to realistic matching problems at CEBAF, and preliminary experimental results are presented.

\section{THE MATCHING SOLUTION}

\subsection{Formulating the problem}

The 4-quadrupole matching problem can be formulated as follows. In the absence of cross-plane coupling, the overall transfer matrices in the two transverse planes, $\mathbf{M x}$ and $\mathbf{M y}$, must satisfy the following conditions

$$
\begin{aligned}
& \mathbf{M}_{\mathrm{X}} \cdot\left(\begin{array}{cc}
\beta_{\mathrm{x}}^{1} & -\alpha_{\mathrm{x}}^{1} \\
-\alpha_{\mathrm{x}} & \gamma_{\mathrm{x}}^{1}
\end{array}\right) \cdot \mathbf{M}_{\mathrm{X}}^{\mathrm{T}}=\left(\begin{array}{cc}
\beta_{\mathrm{x}}^{2} & -\alpha_{\mathrm{x}}^{2} \\
-\alpha_{\mathrm{x}}^{2} & \gamma_{\mathrm{x}}^{2}
\end{array}\right) \\
& \mathbf{M}_{\mathrm{Y}} \cdot\left(\begin{array}{cc}
\beta_{\mathrm{y}}^{1} & -\alpha_{\mathrm{y}}^{1} \\
-\alpha_{\mathrm{y}} & \gamma_{\mathrm{y}}^{1}
\end{array}\right) \cdot \mathbf{M}_{\mathrm{Y}}^{\mathrm{T}}=\left(\begin{array}{cc}
\beta_{\mathrm{y}}^{2} & -\alpha_{\mathrm{y}}^{2} \\
-\alpha_{\mathrm{y}}^{2} & \gamma_{\mathrm{y}}^{2}
\end{array}\right)
\end{aligned}
$$

where the indices 1 and 2 label the initial and end twiss parameters. These 2 transfer matrices in turn are each formed by concatenating the 4 quadrupole matrices (K1 to $\mathrm{K} 4)$ and the matrices representing the 3 intervening sections (A, B, C for $\mathrm{X}$ and $\mathrm{E}, \mathrm{F}, \mathrm{G}$ for $\mathrm{Y}$ ) as follows:

$$
\begin{aligned}
& M_{x}^{\mathrm{K} 1} \cdot M_{x}^{\mathrm{A}} \cdot M_{x}^{\mathrm{K} 2} \cdot M_{x}^{\mathrm{B}} \cdot M_{x}^{\mathrm{K} 3} \cdot M_{\mathrm{x}}^{\mathrm{C}} \cdot M_{x}^{\mathrm{K} 4}=M_{X} \\
& M_{y}^{\mathrm{K} 1} \cdot M_{y}^{\mathrm{E}} \cdot M_{y}^{\mathrm{K} 2} \cdot M_{y}^{\mathrm{F}} \cdot M_{y}^{\mathrm{K} 3} \cdot M_{y}^{\mathrm{G}} \cdot M_{y}^{\mathrm{K} 4}=M_{Y}
\end{aligned}
$$

The problem is thus one of solving $\mathrm{K} 1$ to $\mathrm{K} 4$ given the initial and end twiss parameters and the intervening transfers. Initially the quadrupole matrices will be taken to represent thin lenses, avoiding the need to include transcendental functions in the formulation. Equations (1) and (2) amount to a 4-dimensional algebraic system which, if approached blindly, will soon lead to intractable algebra. A systematic algorithm ensuring clean, step-bystep dimension reduction of the system is therefore critical. The technique developed in [1] was applied to this problem, as briefly described in the next section.

\subsection{Dimension Reduction of the System}

The 4-lens system of (2) is first transformed into a 2lens system consisting of the 2 outer lenses, and a "composite' matrix representing the 2 middle lenses and drift with generic matrix elements. The transformed system is subject to the matching condition (1) to obtain formal solutions for the 2 outer lenses in terms of the twiss parameters, the intervening optics, and the composite matrix. The remaining degrees of freedom of the transformed system, together with the symplectic condition, are used to obtain consistency conditions between the 2 outer lenses and the composite matrix. At this point if the previous formal solutions of the 2 outer lenses are substituted, and the composite matrix is unfolded into its explicit dependencies on the 2 inner lenses, we are left with a system which depends only on the parameters of the 2 inner lenses. In other words,

\footnotetext{
* This work was supported by the U.S. DOE Contract No DE-AC05-84ER40150
} 
systematic decomposition of the constrained degrees of freedom ensures that 2 variables can be cleanly eliminated at each step of the reduction. Since the path leading to this 2-dimensional system is not unique, care must be taken to ensure the most algebraically simple outcome at every branch point.

\subsection{Thin Lens and Semi-Thin Lens Solutions}

The intervening matrices A, B, C, E, F and G in Eq. 2 can be general $2 \times 2$ matrices or pure drifts. The former leads to a $12^{\text {th }}$ order polynomial system for the 2 inner lenses, while the latter an $8^{\text {th }}$ order system. As will be seen, the former can be used to obtain intermediate, or semi-thin lens solutions important to exact thick lens matching.

\section{$2.4 \quad$ Numerical Solution}

With the reduced algebraic system, some powerful numerical algorithms can be called upon for efficient and robust solutions. The algorithm applied in the current case is the Gröbner-basis based NSolve function available in Mathematica 4.0 [2]. This algorithm is particular powerful in its ability to obtain global roots to the polynomial system. This to a large extent eliminates the need for input fine-tuning and ambiguity about existence of real solutions typical of local root-finding algorithms. In certain cases the absence of real global roots can be a valuable indication of system defects or unreasonable input parameters. With rare exceptions where the system may be pathologically conditioned, this function proves to provide very stable global solutions for the 2-dimensional problems, especially in the $8^{\text {th }}$ order case.

Various linear combinations of the two $8^{\text {th }}$ order equations have also been constructed and found complementary to the original equations when numerical conditioning in the latter was less than ideal.

\subsection{Conversion between Thin and Thick Lenses}

The algebraic system corresponding to arbitrary $2 \times 2$ intervening matrices in Section 2.3 can be used in a special case where these matrices are formed by a drift sandwiched between 2 half-thick-lenses, as indicated in Figure 1.

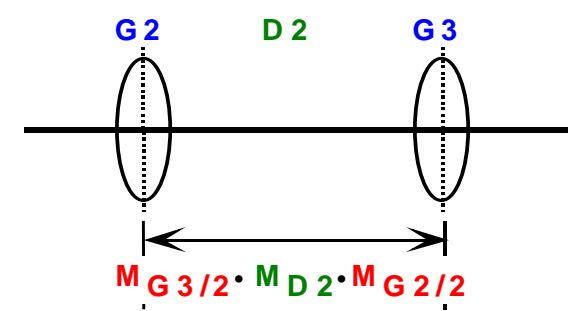

Figure 1: Intervening matrix formed by thick lenses.

The half-thick-lens matrices for the thick lenses G2 and

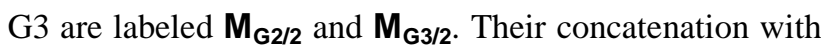
the drift matrix $\mathbf{M}_{\mathbf{D} 2}$ allows us to start with a thick lens system, substitute the concatenated intervening matrices into the algebraic system, and obtain thin lens solutions satisfying the matching condition in addition to the initial thick lenses. These thin lenses are represented by the dotted lines in Figure 1. The solution thus obtained does not correspond to reality, and the thin lens solutions must be "absorbed" into the thick lenses. This is done by algebraically solving the transcendental equations connecting the thin-thick-hybrid lens with a pure thick lens up to the $5^{\text {th }}$ order of small parameter expansion. The resulting formula can be used to convert between these 2 systems with very high accuracy. Iteration can lead to even higher accuracy but is often unnecessary. This method will be referred to as the semi-thin lens solution.

\subsection{The 2-Stage Procedure}

A very effective procedure for obtaining matching solutions involves a 2 -stage process where the pure thin lens solution is obtained first. Being a lower order problem, this solution is often very robust and truly global, with a possibility to select among all returned roots the one with user-desired properties. This solution is "absorbed" into a thick lens system, followed by the application of the semi-thin lens solution, which can be iterated if necessary to produce the desired matching to very high accuracy.

\section{$2.7 \quad$ Numerical Tests}

The algorithm was put through extensive simulated numerical tests and found very effective. In many cases solutions global in nature, as suggested by their drastic deviation from the initial quadrupole values, were found to be more desirable solutions. In most cases without iteration the final pure thick lens solution satisfied all the matching criteria to $10^{-3 \sim 4}$ level. In some cases the algorithm failed to return roots in real number, suggesting configuration flaw or erratic matching conditions. In all cases where real roots could not be found, the same input parameters were tested on standard optics programs such as DIMAD or Optim with no solutions found either. More detailed study looking into zero-contours of the dual polynomial systems indeed confirmed the absence of intersection points in real numbers for such cases. Thus failures to obtain real solutions by the global algorithm can direct attention to systematic problems with more confidence than local algorithms. It is however premature to take the technique in its current state as a fail-safe indicator for systematic problems, as poor numerical conditioning can still obscure the calculation in a very small number of cases.

\section{IMPLEMENTATION OF THE METHOD}

\subsection{Transport Matching at CEBAF}

In the CEBAF accelerator proper where electron beam passes through 2 linacs 5 times each via recirculation arcs, the significance of long range betatron matching lies in the containment of beam size against blowup and tunability on the fixed target. This is in contrast to typical betatron matching where the beam phase space shape is 


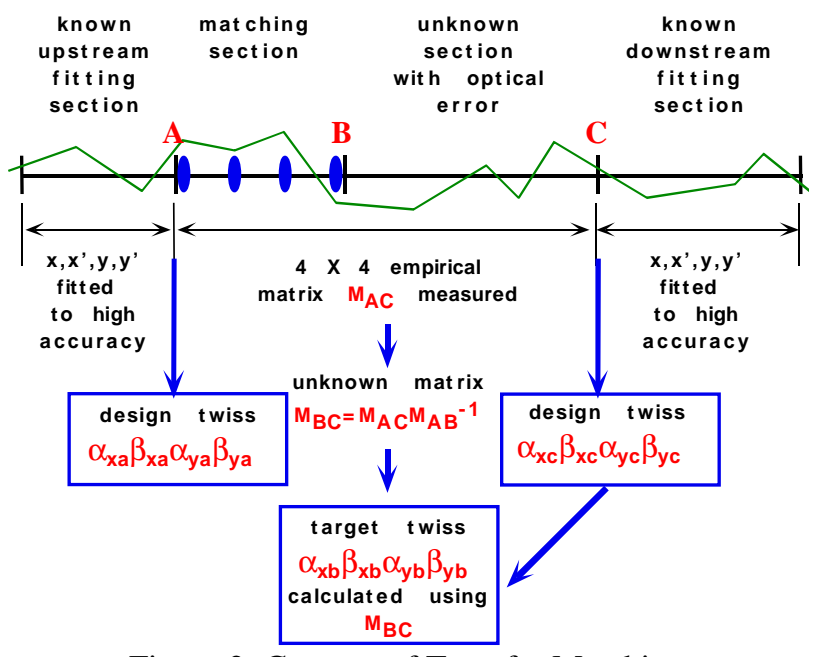

Figure 2: Concept of Transfer Matching

adjusted to match the design. Thus the goal of betatron matching in the CEBAF accelerator proper is to ensure that the transfer matrix of each constituent section is capable of delivering the design twiss parameters to the next section, provided the design twiss parameters are delivered from the previous section. To adapt the matching algorithm to such a task, transfer matrices of each section have to be measured and translated into target twiss parameters. This is demonstrated in Figure 2. The empirical transfer matrix is determined for the section $\mathrm{AC}$ containing the matching quadrupoles, from which the transfer between $\mathrm{B}$ and $\mathrm{C}$ can be inferred. This is then used to determine the target twiss parameters at the end of the matching section, or equivalently, the matching quadrupole changes needed to compensate for the empirical error in the $\mathrm{AC}$ transfer.

\subsection{Transfer Matrix Determination with Symplectic Constraints}

The transfer matrices between $\mathrm{A}$ and $\mathrm{C}$ in Figure 2 is obtained through difference orbits performed routinely on the machine consisting of orbit oscillations covering the phase space in both planes. A Lagrange-multiplier based fitting algorithm was developed to obtain symplectically constrained $2 \times 2$ matrices in both planes. This constraint is necessary because the matching algorithm satisfies the constraints on $\alpha$ and $\beta$, with $\gamma$ taken for granted due to symplecticity. A non-symplectic process results in target $\alpha$ and $\beta$ that, when matched, lead to incorrect $\gamma$.

The symplectic constraint is also seen to have significant correcting effects on the fitted transfer matrices where inadequate signal-to-noise ratio may adversely bias the result.

\section{$3.3 \quad$ On-Line Implementation and Test}

The matching and transfer measurement algorithms have been realized in a combined software package based on $\mathrm{C}++$ and mathematica. A number of on-line tests were performed in March 2000 on the CEBAF accelerator proper. The difference orbits were launched from the beginning of the entire course of the 5-pass recirculation, covering a distance of over $6 \mathrm{~km}$. Each orbit is subject to trajectory fitting in regions with well-controlled optics, and input to the transfer matrix calculation program, which in turn generates empirical target twiss parameters for the matching algorithm. Thus in principle a single set of difference orbits can be used to simultaneously match all constituent sections. The outcome of these tests can be summarized as follows.

- Using the combined RMS of fractional deviations from target $\alpha \mathrm{x}, \beta \mathrm{x}, \alpha \mathrm{y}$ and $\beta \mathrm{y}$ as a measure of success, we could achieve unambiguous, monotonic and sizable reduction of this parameter at every iteration of the process until it is overwhelmed by noise in measurement and quadrupole setting. Typically this parameter starts at a level well over $100 \%$ and is corrected after 2 to 3 iterations to a few percent.

- Causes of failure, in some limited cases, by transfer matrix measurements to produce usable input to the matching algorithm have been identified as, in decreasing order of importance, the loss of orthogonality between difference orbits over long range, uncorrected cross-plane coupling or dispersion, and inadequate local model for trajectory fitting. Remedies or circumventing schemes have been identified and under testing, with some proving effective in preliminary trials.

\section{FUTURE DIRECTIONS}

The program will undergo the following developments and extensions in the near future:

- Algorithmic improvements: Various algorithmic enhancements have been conceived to improve the numerical robustness and efficiency of the method.

- Software improvements: The final on-line application will be made more maintainable.

- Application to beam matching: The technique will be applied to direct beam phase space matching in the CEBAF Injector. Without the complication of target twiss translated from transfer matrix measurement, it is expected that this application will be much more straightforward and successful.

\section{ACKNOWLEDGEMENTS}

The author would like to thank Leigh Harwood and Valeri Lebedev for useful comments, and Alex Bogacz for providing model input files.

\section{REFERENCES}

[1] Y. Chao, J. Irwin, "Solution of a three-thin-lens system with arbitrary transfer properties", SLAC PUB 5834, October 1992.

[2] D. Lichtblau, "Solving finite algebraic systems using numeric Gröbner bases and eigenvalues", Proceedings of the World Conference on Systemics, Cybernetics, and Informatics, Vol 10, 2000, and private communications. 